\title{
Enteric fever caused by Salmonella isolates with decreased susceptibility to ciprofloxacin
}

(Index words: Antibiotic sensitivity, nalidixic acid disc, poor response to fluoroquinolones)

\begin{abstract}
With emerging drug resistance, ciprofloxacin became the frontline antibiotic against Salmonella strains causing enteric fevers worldwide. However, strains with decreased susceptibility to flouroquinolones have recently emerged as a problem in our region. Such strains are not detected by the routine disc diffusion method unless a nalidixic acid disc is also used. They are, however, important clinically since they show poor clinical responses and have higher faecal carriage rates following treatment with fluoroquinolones in usual doses. We report the first two cases of such strains in Sri Lanka, both acquired locally. We recommend the routine use of a nalidixic acid disc in sensitivity testing of Salmonella species, causing enteric fever in laboratories not determining minimum inhibitory concentrations (MICs) for fluoroquinolones in order to detect such strains, so that appropriate clinical decisions regarding antibiotic therapy can be made.
\end{abstract}

\section{Introduction}

Enteric fevers are a group of systemic infections caused by invasive Salmonella species, classically Salmonella typhi but also $S$. paratyphi A, S. shottmulleri (formerly $S$. paratyphi B) and S. hirschfeldii (formerly $S$. paratyphi C) [1]. Chloramphenicol was its firstline therapy for many years until antibiotic resistance became a global problem with the emergence of multi-drug resistant $S$. typhi [2]. Randomised controlled trials have shown that ciprofloxacin is the most effective drug, and resistance rarely emerged during its use. The average fever clearance time was less than 4 days and cure rates over $96 \%$, and less than $2 \%$ developed persistent faecal carriage or relapse [2].

In Sri Lanka, chloramphenicol was considered as the most appropriate antibiotic against $S$. typhi until recently. But $43 \%$ of isolates were resistant to chloramphenicol by 1999 , with increasing resistance to ampicillin, mecillinam and cotrimoxazole, and sensitive to ciprofloxacin and ceftriaxone [3]. In Asia, S typhi strains with decreased susceptibility to fluoroquinolones have become a major problem in recent times [2]. Although these strains were reported as susceptible to ciprofloxacin by the disc diffusion method, they were nalidixic acid-resistant. Their MIC to fluoroquinolones $(0.125-1 \mathrm{mg} / \mathrm{L})$ was 10 times higher than that of fully susceptible strains [2].

We report the first two cases of enteric fever with reduced susceptibility to ciprofloxacin identified by disc diffusion method in Sri Lanka.

\section{Case histories}

S. typhi and S. paratyphi A were isolated from the blood from two patients. Both patients were clinically suspected to have enteric fever, and had no history of travel outside Sri Lanka. Identification was done using standard culture techniques and confirmed serologically. Both patients had shown a poor clinical response to ciprofloxacin. Table 1 shows their antibiotic susceptibility patterns.

Table 1. Antibiotic sensitivity patterns

\begin{tabular}{|c|c|c|c|c|}
\hline \multirow[b]{2}{*}{ Antibiotic } & \multicolumn{2}{|c|}{ Case 1} & \multicolumn{2}{|c|}{ Case 2} \\
\hline & Zone diameter $(\mathrm{mm})$ & Interpretation & Zone diameter $(\mathrm{mm})$ & Interpretation \\
\hline Ampicillin & 26 & Sensitive & 25 & Sensitive \\
\hline Chloramphenicol & 22 & Sensitive & 17 & Resistant \\
\hline Cotrimoxazole & 23 & Sensitive & 23 & Sensitive \\
\hline Ciprofloxacin & 24 & Sensitive & 23 & Sensitive \\
\hline Nalidixic acid & No zone & Resistant & No zone & Resistant \\
\hline Ceftriaxone & 30 & Sensitive & 30 & Sensitive \\
\hline Azithromycin & 19 & Sensitive & 26 & Sensitive \\
\hline
\end{tabular}

Lilani Karunanayake, Senior Registrar in Medical Microbiology and SD Atukorala, Clinical Bacteriologist, National Hospital of Sri Lanka, Colombo.

Correspondence: LK, e-mail: <lilani_k@hotmail.com> (Competing interests: none declared). Received 20 May 2004 and revised version accepted 13 August 2004. 


\section{Discussion}

Decreased susceptibility to fluoroquinolones among Salmonella strains causing enteric fever is a problem for the clinician and the laboratory, unlike fully resistant strains. Laboratories using the disc diffusion method for determining antibiotic sensitivity in vitro may report them as susceptible to fluoroquinolones. But patients infected with them may show a poor clinical response to fluoroquinolones. They may have longer fever clearance time and higher convalescent faecal carriage rate. In addition, they usually show multi-drug resistance [2].

In laboratories where MICs cannot be performed, the concurrent use of a $30 \mu \mathrm{g}$ nalidixic acid disc in disc diffusion antibiotic susceptibility testing is therefore important, until newer criteria for fluoroquinolone resistance evolve. Strains that are nalidixic acid-resistant should be reported as having decreased susceptibility to fluoroquinolones [2]. For such strains, oral azithromycin or higher doses of ciprofloxacin may be given if the patient has uncomplicated enteric fever, or ceftriaxone parenterally in severe cases [2]. Chloramphenicol, amoxicillin or cotrimoxazole can be given if the isolate is fully susceptible and a more effective drug is not available or is unaffordable.

\section{Acknowledgments}

Dr. P Dissanayake and Dr. WK Balasooriya, Physicians, and Ms G Balasooriya, Medical Laboratory Technologist, National Hospital of Sri Lanka, and Dr. C Parry, Consultant Microbiologist at Royal Liverpool University Hospital, UK are gratefully acknowledged for their help.

\section{References}

1. Pearson RD, Guerrant RL. Enteric fever and other causes of abdominal symptoms with fever. In: Mandell, Douglas, and Bennett's Principles and Practice of Infectious Diseases, 5th ed. New York: Churchill Livingstone, 2000: $1136-50$.

2. Parry CM, Hien TT, Dougan G, White NJ, Farrar JJ. Typhoid fever. New England Journal of Medicine 2002; 347: $1770-82$.

3. De Silva N. Bugs versus drugs: who are the champions? The impact of increasing antimicrobial resistance. (Presidential address - section A, 1999). Proceedings of the Sri Lanka Association for the Advancement of Science. Colombo: SLAAS, 2000: Part II, pages 25-40.

\section{Politics and public health in the UK}

Yet these worrying trends receive few column inches in newspapers. Instead, public and political attention on HIV has focused mainly on the demonisation of particular groups, such as asylum seekers and other immigrant communities, and the use of this stigmatised issue to fuel fears and prejudices about immigration.

The UK Government has made statements that suggest it is considering bowing to pressure from anti-immigration lobbies to implement harsher health-screening requirements, including HIV testing, for some people applying to enter the UK. Such measures might quieten public fears and demonstrate apparent Government action on HIV, but they are unlikely to have much effort on the progress and costs of the HIV/AIDS epidemic in the UK.

L Power. HIV and sexual health in the UK: politics and public health. Lancet 2004; 364: 108-9. 